366 L'ORIGINE DE L'INDUSTRIE DE L'OR EN AMÉRIQUE

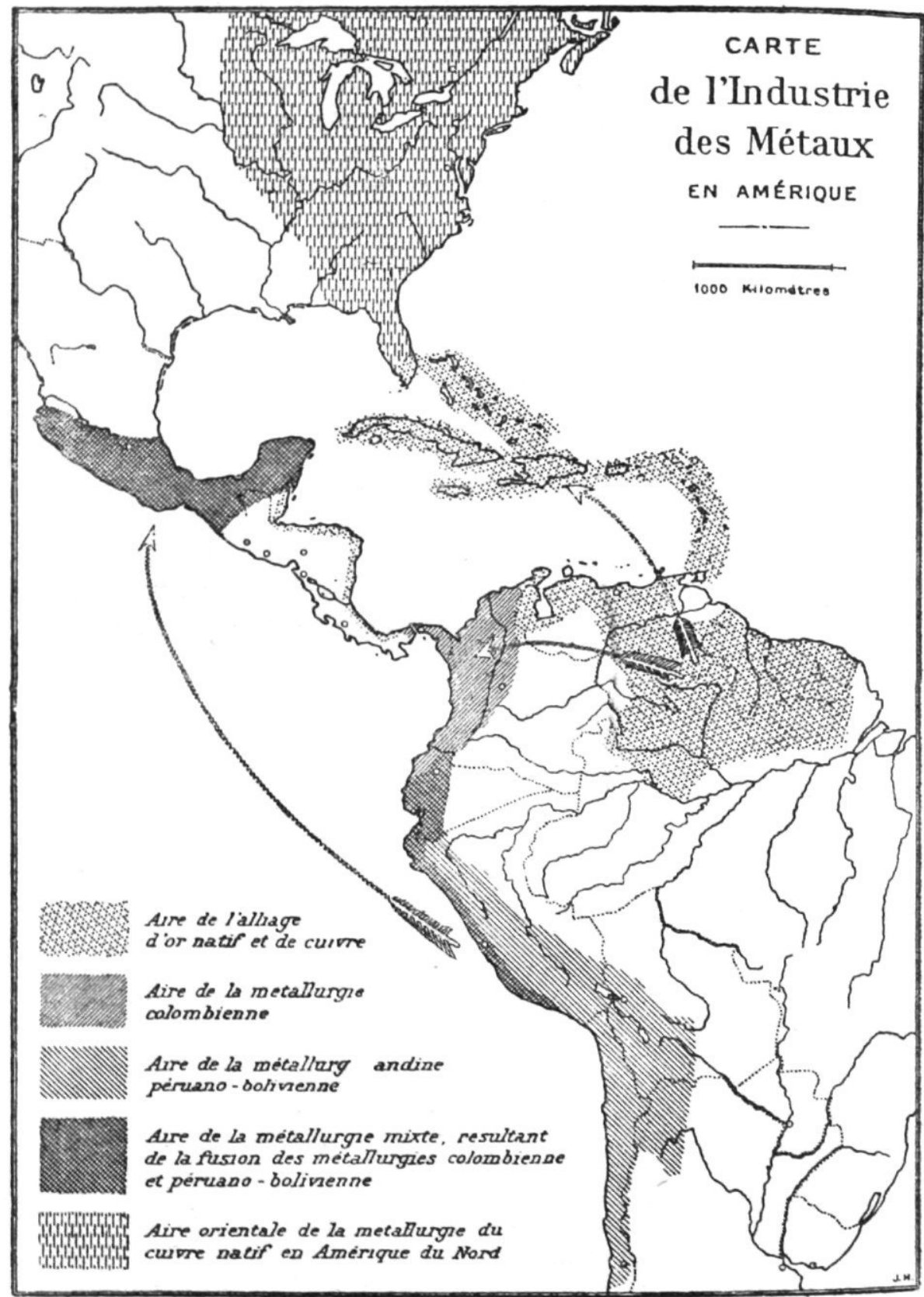




\title{
L'ORIGINE DE L'INDUSTRIE DE L'OR EN AMERIQUE
}

\author{
PAR \\ P. RIVET
}

La région américaine, où l'industrie de l'or a atteint sans contredit son plus grand développement à l'époque précolombienne est celle qui correspond actuellement à la Colombie et à la République de Panamá. Dans tout ce vaste territoire, les tombes aborigènes sont si riches en objets d'or que la recherche et la fouille de ces sépultures $\mathrm{y}$ constituent une profession répandue et assez souvent lucrative ${ }^{1}$ ).

Dans ce domaine, les produits de l'art préhispanique ne sont pas partout les mêmes. On peut en effet y distinguer aisément trois industries assez différentes au point de vue morphologique: l'industrie d'Antioquia dont le type nous est fourni par les produits des anciens Quimbaya, l'industrie du haut plateau de Bogotá, qui est celle des anciens Chibcha, et l'industrie du Chiriquí dans l'isthme de Panamá. Mais, malgré les différences morphologiques très nettes qui existent entre ces trois industries, l'étude de la technique des orfèvres montre que les procédés employés étaient partout sensiblement les mêmes et certainement fondamentalement identiques. Partout en effet, les artistes précolombiens utilisaient abondamment l'or natif plus ou moins argentifère de leur propre pays, soit seul, soit associé en proportion variable avec le cuivre, et savaient mettre en couleur les alliages à bas titre, c'est-à-dire leur donner, par l'action combinée d'un acide et du feu, une cou-

\footnotetext{
1) Arango C. (Luis). Recuerdos de la guaqueria en el Quindio. Barcelone, s.d., [1923].
} 


\section{L'ORIGINE DE L'INDUSTRIE DE L'OR EN AMÉRIQUE}

leur correspondant à un alliage plus riche en or ; ils connaissaient le tréfilage, le laminage, le placage de l'or sur le cuivre, le repoussage, le coulage ordinaire et à la cire perdue, et savaient pratiquer la soudure autogène. Bien qu'ils connussent le cuivre, ils l'utilisaient relativement peu à l'état pur et ont toujours ignoré le bronze et l'argent ${ }^{\mathbf{1}}$ ).

Sur ce fonds commun de connaissances techniques se sont développées les trois industries à facies différents, que j'ai signalées plus haut, tel procédé étant employé plus fréquemment ici que là, mais sans que l'unité originelle de tout cet art puisse échapper à une étude un peu attentive.

L'industrie colombienne de l'or a débordé largement au delà des frontières de l'actuelle République de Colombie; elle a été transportée dans les régions méridionales par les invasions de tribus appartenant à la famille linguistique chibcha, et même s'est répandue par la voie de l'emprunt au-delà des limites que la linguistique permet de fixer actuellement à ces migrations. J'ai montré en effet que toute la vaste contrée comprenant la République de l'Équateur et la côte péruvienne avait subi, soit directement, soit indirectement, l'influence de la technique métallurgique colombienne ${ }^{2}$ ). Dans ces dernières régions, une indus-

1) Max Uhle a contesté récemment cette dernière conclusion (Boletin de la Academia nacional de historia. Quito, t. VII, 1923, p. 271). Les faits sont cependant indéniables. Sur plus d'un millier d'objets métalliques de Colombie décrits à ce jour, il n'y a que deux objets en argent que j'ai décrits moi-même, et il s'agit d'objets dont la provenance exacte n'est pas connue. Achetés à Bogotá, ils peuvent y avoir été importés, soit à une époque ancienne, soit même à une époque récente, de l'Équateur où l'industrie de l'argent était connue. C. M. Larrea croit qu'il s'agit d'ex-voto de la période coloniale provenant de l'ancien sanctuaire de San Jacinto, à Yaguachi (Boletin de la Sociedad ecuatoriana de estudios históricos americanos. Quito, t. IV, 1920, p. 521). Je suis actuellement convaincu de l'exactitude de sa remarque.

2) Créqui-Montfort (G. de), Rivet (P.) et Arsandaux (H.). Contribution à l'étude de l'archéologie et de la métallurgie colombiennes. Journal de la Société des Américanistes de Paris, n.elle série, t. XI, 1914-1919, p. 523591; Rivet (Paul). Note complémentaire sur la métallurgie sud-américaine. Journal de la Société des Américanistes de Paris, n. elle série, t. XIII, 1921, p. 233-238; Arsandaux (H.) et Rivet (P.). L'orfèvrerie du Chiriqui et de Colombie. Journal de la Société des Américanistes de Paris, n.ello série, t. XV, 1923, p. $169-182$. 
trie métallurgique toute différente, née sur le haut plateau péruano-bolivien, est venue se superposer à la métallurgie colombienne pour constituer une industrie mixte utilisant à la fois le cuivre, l'or, l'étain, l'argent, exceptionnellement le plomb, et les divers alliages de ces métaux, et en possession d'une technique complète remarquablement évoluée. J'ai établi en outre que, selon toute vraisemblance, cette industrie mixte avait été transportée au Mexique, toute constituée et à une date relativement récente, par les navigateurs qui trafiquaient tout le long de la côte du Pacifique ${ }^{\mathbf{1}}$ ).

Malgré le développement remarquable que le travail de l'or a pris aux mains des peuples chibcha, je ne crois pas que ces peuples en aient été les inventeurs. A mon sens, leur rôle se serait limité à perfectionner et à diffuser une technique dont les éléments essentiels leur auraient été apportés d'ailleurs. Je ne puis revenir ici sur les détails d'une démonstration que j'ai exposée dans un long mémoire ${ }^{2}$ ). Je me contenterai de rappeler les principaux faits sur lesquels j'ai cru pouvoir m'appuyer.

A l'époque où les Espagnols arrivèrent en Amérique, ils trouvérent, entre les mains des indigènes des Antilles et de toute la vaste région qui s'étend au nord de l'Amazone et comprend les Guyanes et le Vénézuéla, des objets fabriqués avec un alliage d'or natif et de cuivre, que les Arawak appelaient guanin et les Karib karakoli, alliage absolument identique à celui qu'utilisaient les aborigènes

\footnotetext{
1) Arsandaux (H.) et Rivet (P.). Étude sur l'archéologie mexicaine. Académie des Inscriptions et Belles-Lettres. Comptes rendus des séances de l'année 1921. Paris, p. 337-340; Recherches sur la métallurgie mexicaine. L'Anthropologie. Paris, t. XXXI, 1921, p. 521-522; Contribution d l'étude de la métallurgie mexicaine. Journal de la Société des Américanistes de Paris, nouv. série, t. XIII, 1921, p. 261-280; Nouvelle note sur la métallurgie mexicaine. L'Anthropologie. Paris, t. XXXIII, 1923, p. 63-85.

$\left.{ }^{2}\right)$ Rivet (P.). L'orfèvrerie précolombienne des Antilles, des Guyanes, et du Vénézuéla dans ses rapports avec l'orfèvrerie des autres régions américaines. Journal de la Société des Américanistes de Paris, nouv. série, t. XV, 1923, p. $183-213$.
} 


\section{L'ORIGINE DE L'INDUSTRIE DE L'OR EN AMÉRIQUE}

colombiens. Or, s'il est à peu près certain qu'aucune migration chibcha n'a jamais atteint le bassin de l'Orénoque et les Guyanes, nous avons la preuve que des Karib ont autrefois envahi le haut plateau colombien, où ils ont encore des représentants, et nous savons par les anciens chroniqueurs que le mot karakoli (sous la forme karikuri) a pénétré lui aussi dans cette région. On a donc d'excellentes raisons de supposer que ce sont les Karib qui ont apporté aux Chibcha les éléments de la technique du travail de l'or. Le centre primitif de découverte de cette technique doit donc être reporté dans l'arrière-pays guyanais, précisément dans cette région où la légende situait le fameux El Dorado, ce pays aux richesses prodigieuses que tant d'aventuriers s'efforcèrent en vain d'atteindre. Une fois de plus, à la base d'une légende, on trouve un fait exact que l'imagination des hommes s'est complue à déformer en l'amplifiant.

Cette migration karib est certainement très ancienne; en effet, l'industrie de l'or colombienne, à laquelle elle a donné naissance et que les peuples chibcha ont répandu ensuite vers le sud, apparaît sur la côte péruvienne dès l'époque proto-chimú, qui représente un des premiers horizons de la civilisation péruvienne, dont Max Uhle place le début au 2ème siècle de notre ère. Je ne cite d'ailleurs cette date qu'à titre d'indication, car je suis d'avis que nous manquons d'éléments jusqu'à présent pour établir en Amérique du Sud une chronologie absolue sérieuse et qu'il est préférable de se contenter d'une chronologie relative qui paraît actuellement reposer sur des bases assez solides. 